\title{
RbAp48, a novel inhibitory factor that regulates the transcription of human immunodeficiency virus type 1
}

\author{
JUAN WANG ${ }^{1,2^{*}}$, JIN YANG $^{3,5^{*}}$, ZONGXING YANG $^{4}$, XIANGYUN LU $^{1,2}$, \\ CHANGZHONG JIN ${ }^{1,2}$, LINFANG CHENG ${ }^{1,2}$ and NANPING WU ${ }^{1,2}$
}

\begin{abstract}
${ }^{1}$ State Key Laboratory for Diagnosis and Treatment of Infectious Diseases, The First Affiliated Hospital, College of Medicine, Zhejiang University; ${ }^{2}$ Collaborative Innovation Center for Diagnosis and Treatment of Infectious Diseases, Hangzhou, Zhejiang 310003; ${ }^{3}$ Department of Medicine, Blood Center of Zhejiang Province, Hangzhou, Zhejiang 330100; ${ }^{4}$ Xixi Hospital of Hangzhou, Hangzhou, Zhejiang 310023, P.R. China
\end{abstract}

Received October 31, 2015; Accepted May 10, 2016

DOI: $10.3892 /$ ijmm.2016.2598

\begin{abstract}
Retinoblastoma binding protein 4 (RbAp48) is a histone chaperone which has been suggested to play a role in gene silencing. However, the role of RbAp48 in human immunodeficiency virus type 1 (HIV-1) infection and gene replication has not been determined to date, to the best of our knowledge. For this purpose, we demonstrated in the present study that RbAp48 expression was upregulated by HIV-1 infection, whereas the knockdown of RbAp48 promoted HIV infection and the production of virus particles. The ectopic expression of RbAp48 inhibited HIV-1 expression, and this inhibition correlated with a marked decrease in the expression of HIV-1 genomic RNA and various RNA transcripts. Further experiments to determine the mechanism responsible for the inhibitory effects of RbAp48 revealed that the ectopic expression of RbAp48 repressed HIV-1 long terminal repeat (LTR)-mediated basal transcription as well
\end{abstract}

Correspondence to: Professor Nanping Wu, State Key Laboratory for Diagnosis and Treatment of Infectious Diseases, The First Affiliated Hospital, College of Medicine, Zhejiang University, 79 Qingchun Road, Hangzhou, Zhejiang 310003, P.R. China E-mail: flwnp2013@163.com

Present address: ${ }^{5}$ Center for Translational Medicine, The Affiliated Hospital of Hangzhou Normal University, Hangzhou, Zhejiang 330100, P.R. China

\section{*Contributed equally}

Abbreviations: RbAp48, retinoblastoma binding protein 4; LTR, long terminal repeat; ChIP, chromatin immunoprecipitation; EMSA, electrophoretic mobility shift assay; HDAC, histone deacetylase; CAF-1, chromatin assembly factor 1 ; NuRD, nucleosome remodelling and histone deacetylase complex; ELISA, enzyme-linked immunosorbentassay;MOI,multiplicity of infection;GAPDH, glyceraldehyde3-phosphate dehydrogenase; NURF, nucleosome-remodelling factor; PRC, polycomb repressive complex

Key words: HIV, retinoblastoma binding protein 4, repression, long terminal repeat, transcription as TNF- $\alpha$ - and phorbol 12-myristate 13-acetate (PMA)-activated transcription. Furthermore, the results of the electrophoretic mobility shift assay (EMSA) and chromatin immunoprecipitation (ChIP) analysis revealed that RbAp48 binds to the HIV-1 LTR in vitro. Taken together, these findings demonstrate that, as a transcriptional cofactor, $\mathrm{RbAp} 48$ is likely to act as a potent antiretroviral defense.

\section{Introduction}

The replication of human immunodeficiency virus type 1 (HIV-1) is a complex event mediated by a dynamic interplay between the host and viral factors (1). During the HIV-1 life cycle, HIV DNA is integrated into the host genome, resulting in proviral DNA. The transcription of the integrated provirus is essential for viral gene replication and the production of viral particles. The HIV-1 5' long terminal repeat (LTR), as a promoter of the integrated provirus, comprises elements that regulate HIV-1 transcription (2). HIV gene expression may be driven by host transcription factors whereas HIV transcriptional silencing is promoted by an inactive state of chromatin (3), which is regulated by transcriptional inhibitors and chromatin remodelling factors. In this regard, chromatin organization plays an important role in regulating HIV-1 gene expression, as it affects the accessibility of transcription factors to cis-elements on the HIV-1 5' LTR (4).

As a transcription cofactor, retinoblastoma binding protein 4 (RbAp48) was first identified by Qian et al (5). It is a $48-\mathrm{kDa}$ protein which is closely combined with the retinoblastoma protein $(\mathrm{Rb})$. Owing to its ability to directly interact with the histones H3-H4 (6), RbAp48 is the key subunit of complexes that target chromatin remodelling factors to their histone and DNA substrates. RbAp48 is present in the chromatin assembly factor 1 (CAF-1) complex (7), which plays a role in chromatin assembly. RbAp48 is a subunit of the nucleosome remodelling and histone deacetylase complex (NuRD) (8), which suppresses transcription by removing the acetyl groups on histones and by nucleosome remodelling. RbAp48 is also part of co-repressor complexes, such as the core histone deacetylase (HDAC) complex (9). Notably, several components of these chromatin remodelling complexes, such as the NuRD and HDAC complexes, are known to interact with the HIV-1 LTR (10). 
Although its role as a histone chaperone is perhaps the best studied of its functions, RbAp48 is involved in other important cellular processes. For example, RbAp48 is required to maintain multiple human pluripotent stem cell types by modifying the $\mathrm{Rb}-\mathrm{E} 2 \mathrm{~F}$ pathway (11). Additionally, through an interaction with oestrogen receptor (ER) $\alpha$ that limits the expression of oestrogen-responsive genes (12), RbAp48 induces apoptosis in specific tissues, depending on the level of oestrogen deficiency. Autoimmune exocrinopathy may be induced by the overexpression of RbAp48 (13). As a component of the HDAC complex recruited by $\mathrm{Rb}, \mathrm{RbAp} 48$ has been implicated in transcriptional repression (14). Taken together, the various functions of RbAp48 suggest that it plays important roles in the regulation of gene transcription.

Gene transcription is an important step in HIV infection. However, the interrelationship between RbAp48 and HIV infection has not yet been established. In the present study, we demonstrated that RbAp48 inhibits the production of viral particles at the transcriptional level. Promoter activity testing provided evidence that RbAp48 inhibits basal as well as TNF- $\alpha$ and PMA-activated transcription through direct binding to the HIV-1 LTR. Taken together, these findings demonstrated, for the first time to the best of our knowledge, that RbAp48 harbours antiviral activity, and supports the hypothesis that RbAp48 may exert a negative regulatory effect on HIV-1 transcription.

\section{Materials and methods}

Plasmid constructs. The generation of the luciferase (Luc) reporter construct pGL3-LTR-Luc and the serial truncation reporter constructs were performed as previously described (15). The viral molecular clone pNL4-3 was kindly provided by Dr Y.-J. Wang (Zhejiang University, Hangzhou, China). The pRbAp48 vector and control pCTL clone were obtained from OriGene Technologies, Inc., (Rockville, MD, USA). The following short hairpin RNA (shRNA) directed against RbAp48 were used as previously described: sense, 5'-CGAGGAAUACAAAAUAUG GTT-3' and antisense, 5'-CCAUAUUUUGCUCGTT-3' (13). The sh-RbAp48 vector based on a pGP-U6 backbone was synthesized by Gene Pharma (Shanghai, China) and sh-NC was used as a control. The vector psh-NC (sh-NC) was constructed based on pGP-U6 backbone with an irrelevant short hairpin sequence.

Cell culture and transfections. The 293T cells (ATCC; Manassas, VA, USA) and TZM-bl cells were cultured in DMEM supplemented with $10 \%$ fetal bovine serum (FBS; Life Technologies, Carlsbad, CA, USA) and penicillin-streptomycin (Life Technologies). The CEM-ss cells were maintained in RPMI supplemented with $10 \% \mathrm{FBS}, 100 \mathrm{U} / \mathrm{ml}$ penicillin, $100 \mu \mathrm{g} / \mathrm{ml}$ streptomycin, 0.1 M HEPES and $2 \mathrm{mM}$ glutamine at $37^{\circ} \mathrm{C}$. The TZM-bl and CEM-ss cells were kindly provided by Dr Paul Zhou (16). Transfections were performed with the indicated plasmids using Lipofectamine 2000 (Life Technologies). The Amaxa Human T-cell Nucleofector kit was used to transfect the CEM-ss cells using the Amaxa Nucleofector II device (both from Amaxa, Cologne, Germany).

Production of viral stocks and infection. Viral stocks were produced using H9/HTLV-IIIB (NIH 1983) cells (kindly provided by Dr Y.-J. Wang, Zhejiang University, Hangzhou, China), as previously described (17). Viral p24 antigens were detected using an enzyme-linked immunosorbent assay (ELISA) kit (Wantai Bio-Pharm, Beijing, China and ImmunoDX LLC, Woburn, MA, USA). The CEM-ss cells were cultured at a density of $2.0 \times 10^{6} /$ well and infected with HIV-1 at a multiplicity of infection (MOI) of 1 by spinoculation at $1,200 \times \mathrm{g}$ for $2 \mathrm{~h}$ at $25^{\circ} \mathrm{C}(18)$. The cells were centrifuged at $300 \mathrm{x} \mathrm{g}$ for $5 \mathrm{~min}$ to remove free virus. The quantification of infectious viruses was performed by infecting TZM-bl cells, as previously described (19). The mock-infected cells indicated cells, which were not infected with viral stocks. In order to accurately compare with the cells infected with viral stocks, the mock-infected cells were also centrifuged by spinoculation at $1,200 \mathrm{x} g$ for $2 \mathrm{~h}$ at $25^{\circ} \mathrm{C}$ as the cells infected with viral stocks.

Luciferase assay and stimulation. The $293 \mathrm{~T}$ cells were seeded $\left(2 \times 10^{4}\right.$ cells/well) and cultured overnight. The cells were transiently transfected with a firefly luciferase construct along with a pRbAp48 construct and a Renilla luciferase plasmid. As negative controls, transfections were performed with the pGL3Basic vector (Promega Corp., Madison, WI, USA) and the empty pCTL vector (OriGene Technologies, Inc.), respectively. Additionally, the TZM-bl cells were seeded and treated as the indicated steps. The cells were stimulated with $25 \mathrm{ng} / \mathrm{ml}$ phorbol 12-myristate 13-acetate (PMA; Sigma-Aldrich, St. Louis, MO, USA) and $20 \mathrm{ng} / \mathrm{ml} \mathrm{TNF-} \alpha$ (R\&D Systems, Minneapolis, MN, USA). At $48 \mathrm{~h}$ after transfection, luciferase activity was determined according to the manufacturer's instructions (Promega Corp.).

Reverse transcription-quantitative polymerase chain reactionx (RT-qPCR). Total cellular RNA was extracted using TRIzol reagent (Life Technologies). RNA aliquots (200 ng) were reverse transcribed using a PrimeScript RT reagent kit (Takara Bio, Inc., Otsu, Japan). Quantitative detection of HIV-1 DNA was performed using primers spanning the LTR-gag region (20). HIV-1 mRNA species were determined as previously described $(21,22)$. All primers used are presented in Table I. Amplification was performed using a C1000 Thermal Cycler detection system (Bio-Rad Laboratories, Inc., Hercules, CA, USA) in triplicate using $1 \mathrm{ng}$ of cDNA, $125 \mathrm{nM}$ forward and reverse primer and $25 \mu \mathrm{l} 2 \mathrm{X} \mathrm{SYBR}{ }^{\circledR}$ Premix $\mathrm{Ex} \mathrm{Taq}^{\mathrm{TM}}$ (Takara Bio, Inc) in a $50 \mu \mathrm{l}$ reaction. The reaction parameters were $95^{\circ} \mathrm{C}$ for $1 \mathrm{~min}$, followed by 42 cycles of $95^{\circ} \mathrm{C}$ for $15 \mathrm{sec}, 56^{\circ} \mathrm{C}$ for $25 \mathrm{sec}$, and $72^{\circ} \mathrm{C}$ for $30 \mathrm{sec}$.

Western blot analysis. The cells were treated and collected at the indicated time-points and lysed using cell lysis buffer (Cell Signaling Technology, Inc., Danvers, MA, USA) supplemented with phenylmethanesulfonyl fluoride (Cell Signaling Technology, Inc.). Proteins were quantitated using the BCA Assay kit (Thermo Fisher Scientific, Inc., Pittsburg, PA, USA). Proteins were fractioned by SDS-PAGE through $10 \%$ denaturing acrylamide gels, transferred to polyvinylidene fluoride membranes and detected with a monoclonal antibody directed against RbAp48 (ab79416; Abcam, Burlingame, CA, USA), or glyceraldehyde-3-phosphate dehydrogenase (GAPDH) (D16H11; Cell Signaling Technology, Inc.). Horseradish peroxidase-conjugated secondary anti- 
Table I. Primers used in the present study.

\begin{tabular}{|c|c|c|}
\hline Name & Primer sequences & HIV-1 locatior \\
\hline RBBP4 & $\begin{array}{l}\text { 5'-ACCCTGGCTAGAGATGCAGA-3' } \\
\text { 5'-CCTTTTCCCCTGCTTTGTATCG-3' }\end{array}$ & \\
\hline GAPDH & $\begin{array}{l}\text { 5'-AGCCACATCGCTCAGAACAC-3' } \\
\text { 5'-GAGGCATTGCTGATGATCTTG-3' }\end{array}$ & \\
\hline PBS-LTR & $\begin{array}{l}\text { 5'-TAGCAGTGGCGCCCGA-3' } \\
\text { 5'-TCTCTCTCCTTCTAGCCTCCGC-3' }\end{array}$ & $\begin{array}{l}630-645 \\
769-790\end{array}$ \\
\hline Unspliced RNA & $\begin{array}{l}\text { 5'-GACGCTCTCGCACCCATCTC-3' } \\
\text { 5'-CTGAAGCGCGCACGGCAA-3' }\end{array}$ & \\
\hline Single-spliced RNA & $\begin{array}{l}\text { 5'-GGCGGCGACTGGAAGAAGC-3' } \\
\text { 5'-CTATGATTACTATGGACCACAC-3' }\end{array}$ & \\
\hline Multiple-spliced RNA & $\begin{array}{l}\text { 5'-GACTCATCAAGTTTCTCTATCAAA-3' } \\
\text { 5'-AGTCTCTCAAGCGGTGGT-3' }\end{array}$ & \\
\hline \multicolumn{3}{|l|}{ ChIP primer } \\
\hline LTR promoter & $\begin{array}{l}\text { 5'-AGGTTTGACAGCCGCCTA-3' } \\
\text { 5'-AGAGACCCAGTACAGGCAAAA-3' }\end{array}$ & $\begin{array}{l}261-278 \\
441-461\end{array}$ \\
\hline GAPDH promoter & $\begin{array}{l}\text { 5'-GGACCTGACCTGCCGTCTAGAA-3' } \\
\text { 5'-GGTGTCGCTGTTGAAGTCAGAG-3' }\end{array}$ & \\
\hline
\end{tabular}

RBBP4, retinoblastoma binding protein 4; LTR, long terminal repeat; ChIP, chromatin immunoprecipitation; HIV-1, human immunodeficiency virus type 1 .

bodies (1:2000; \#7074; Cell Signaling Technology, Inc.) and enhanced chemiluminescence reagents (Amersham, Piscataway, NJ, USA) were used to visualize the proteins.

Electrophoretic mobility shift assay (EMSA). The TZM-bl cells were stimulated with $20 \mathrm{ng} / \mathrm{ml} \mathrm{TNF}-\alpha$ for $24 \mathrm{~h}$ in 6 -well plates for the indicated times. Nuclear extracts was harvested using the NE-PER cytoplasmic and nuclear extraction reagents kit (Thermo Fisher Scientific, Inc.) according to the manufacturer's instructions. The probes were labelled with biotin at the $3^{\prime}$ and $5^{\prime}$ ends and they were annealed double-stranded oligonucleotides. The sequence of the probe corresponding to NF- $\kappa \mathrm{B}$ binding elements on the LTR was as follows: TTGCTACAAGGGACTTTCCGCTGGGGACTT TCCAGTTAGTC. The mutated probe was as follows: TTGC

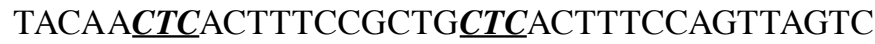
(the mutation sites are in italic bold letters and underlined). EMSAs were performed using a LightShift Chemiluminescent EMSA kit (Thermo Fisher Scientific, Inc.). Wild probes were incubated with $10 \mu \mathrm{g}$ of nuclear extracts or not at room temperature for $30 \mathrm{~min}$ in $15 \mu \mathrm{l}$ reaction buffer. The reaction buffer was a mixture containing $1 \mathrm{X}$ binding buffer, $2.5 \%$ glycerol, $5 \mathrm{mM} \mathrm{MgCl}, 50 \mathrm{ng} / \mu \mathrm{l}$ poly(dI.dC) and $0.05 \%$ NP-40. For competitor assays, a 40-fold molar excess of unlabelled, double-stranded probe was included in the binding reactions. The mutated probes were used to verify the specificity of shift and an anti-RbAp48 antibody was used for a supershift. After $3.75 \mu \mathrm{l}$ of loading buffer was added to all binding reactions, the binding reactions were electrophoresed in $6 \%$ polyacrylamide gel at $100 \mathrm{~V}$ for $60 \mathrm{~min}$ in $0.5 \mathrm{X}$ TBE buffer. The gel and a positively charged nylon membrane were sandwiched in a transfer unit and transferred at $380 \mathrm{~V}$ for $60 \mathrm{~min}$ in cooled $0.5 \mathrm{X}$ TBE. The probes in the membrane were blocked with blocking buffer for $15 \mathrm{~min}$, incubated with streptavidinhorseradish peroxidase conjugate (1:300 Thermo Fisher Scientific, Inc.) for $15 \mathrm{~min}$, washed with $1 \mathrm{X}$ washing buffer four times, equilibrated with the substrate equilibration buffer for $5 \mathrm{~min}$, incubated with substrate working solution and finally, visualized using a VersaDoc MP 5000 imaging system (obtained from Bio-Rad Laboratories, Inc.).

Chromatin immunoprecipitation (ChIP) analysis. ChIP analysis was performed in accordance with the manufacturer's instructions for the ChIP assay kit (Millipore Corp., Billerica, MA, USA). Briefly, the TZM-bl cells were seeded in a 6-well plate and cross-linked with $1 \%$ formaldehyde for $10 \mathrm{~min}$ at room temperature. Glycine $(0.125 \mathrm{M})$ was added into the wells to quench the unreacted formaldehyde. The cells were washed, scraped and pelleted with cooled PBS containing protease inhibitor cocktail II. SDS lysis buffer (1\%) was used to lyse the cell pellets. Sonication of the lysate was conducted on wet ice. An aliquot of the sheared lysate was saved as a control for the amount of input DNA at $4^{\circ} \mathrm{C}$ until elution. The remainder was diluted with dilution buffer and incubated with RbAp48 antibody overnight at $4^{\circ} \mathrm{C}$. Protein A-agarose was used to capture the immunocomplexes. Elution buffer including $1 \%$ SDS and $100 \mathrm{mM} \mathrm{NaHCO}$ eluted the immunocomplexes from the beads. The eluant was reverse cross-linked at $65^{\circ} \mathrm{C}$ overnight. After DNA purification, RbAp48 enrichment in specific DNA was analysed by qPCR. The primers for amplication are presented in Table I. Anti-normal human immunoglobulin G was used for the negative control. 
A

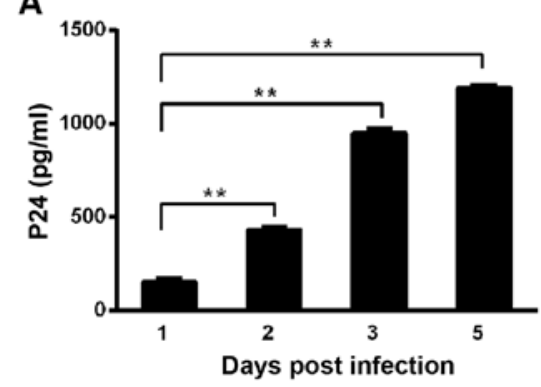

C

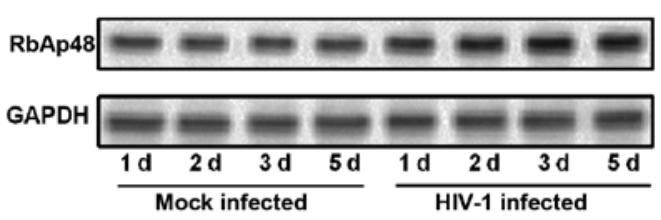

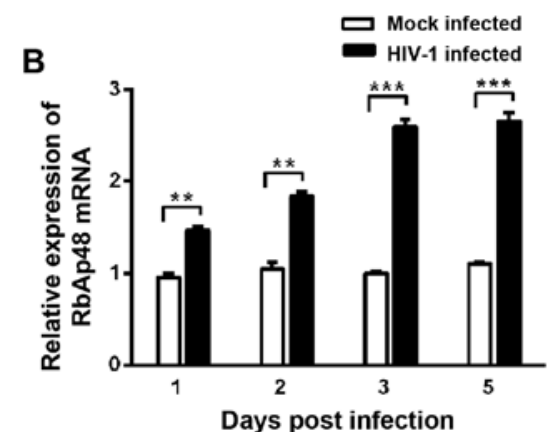

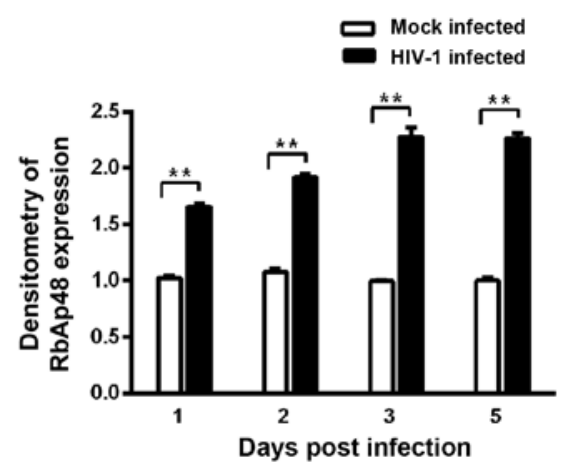

Figure 1. HIV-1 infection induces intracellular retinoblastoma binding protein 4 (RbAp48) expression. (A) CEM-ss cells were infected with HIV-1 stocks. The p24 level in culture supernatants at the indicated time was quantified by enzyme-linked immunosorbent assay (ELISA). (B) RbAp48 mRNA expression in HIV1-infected CEM-ss cells. Quantification of GAPDH was used to normalize the variations between samples. (C) RbAp48 protein expression in HIV-1 infected or mock infected cells (left panel). In the right panel, band intensities were measured by densitometry and normalized to GAPDH. ${ }^{* *} \mathrm{P}<0.01$ and ${ }^{* * * *} \mathrm{P}<0.001$.

Statistical analysis. Each experiment was performed 3 times independently. Data are presented with the means \pm SEM. An unpaired two-tailed student's t-test was used to compare data between two groups. $\mathrm{P}<0.05$ was considered to indicate a statistically significant difference (GraphPad Software, Inc., La Jolla, CA, USA).

\section{Results}

HIV-1 infection induces intracellular RbAp48 expression. To explore the effects of RbAp48 during the early stages of viral infection, we examined the expression of endogenous RbAp48 in cells infected with HIV-1. CEM-ss cells, a CD4 ${ }^{+}$T-cell line, were infected with viral stock. Increasing expression of HIV-1 p24 antigen was observed in the culture supernatants post infection (Fig. 1A). Concomitantly, the expression of RbAp48 mRNA was increased by 1.85 -fold at 2 days post-infection, and it was increased by 2.6 -fold at 3 days post-infection (Fig. 1B), respectively, as compared with the expression level determined from the mock-infected cells at the same time points. Furthermore, we evaulated the protein expression of RbAp48 using western blot analysis. RbAp48 protein expression increased significantly at 1 day after HIV-1 infection, and was elevated up to 2.3-fold at 5 days after HIV-1 infection, as shown by western blot analysis (Fig. 1C, left panel) and densitometry (Fig. 1C, right panel).

RbAp48 inhibits HIV-1 expression at the transcriptional level. To address the question of whether induced RbAp48 expression may influence HIV-1 infection, the CEM-ss cells were transfected with an shRNA vector targeting RbAp48 (sh-RbAp48) or its control (sh-NC) by electroporation and infected with HIV-1 at 1 day post-transfection. The confirmation of knockdown efficiency was performed by western blot analysis (Fig. 2A). We observed a 2.14 \pm 0.3 -fold increase in viral p24 levels in the supernatant at 1 day post-infection compared with those in the control (Fig. 2B, left panel). Quantification of infectious viruses from the supernatant by infecting TZM-bl indicator cells revealed a $1.71 \pm 0.22$-fold increase (Fig. $2 \mathrm{~B}$, right panel). These results indicate that $\mathrm{RbAp} 48$ negatively regulates the production of HIV viral particles and the RbAp48 depletion relieves this repressive effect.

Next, we evaluated the production level of HIV-1 particles in the presence of exogenous RbAp48. The 293T cells were transfected with a constant amount of pNL4-3 together with increasing concentrations of RbAp48 expression construct (pRbAp48) or control vector (pCTL). As shown in Fig. 2C, increasing amounts of RbAp48 inhibited HIV-1 production. As a transcription cofactor, RbAp48 may influence HIV-1 transcription directly (23). To further examine this issue, we used absolute quantification to monitor the viral load produced in the infected cells overexpressing RbAp48. As shown in Fig. 2D, RbAp48 overexpression results in a 1.17- to 5.18-fold decrease in HIV-1 genomic RNA levels, which correlated with a decrease in the viral p24 levels or the production of infectious viruses in the supernatant (Fig. 2C).

HIV-1 mRNA species are produced by alternative splicing of the primary viral RNA transcript (24), and the ongoing production of virus by cells latently infected with HIV-1 produces both unspliced and multiple-spliced RNAs. RbAp48 may be part of pre-mRNA processing or splicing complexes (25); therefore, we detected specific types of spliced RNA in cells overexpressing RbAp48. As shown in Fig. 2E-G, when values were normalized to GAPDH mRNA levels, RbAp48 inhibited multiple-, single- or unspliced HIV-1 RNA to similar extents. 
A

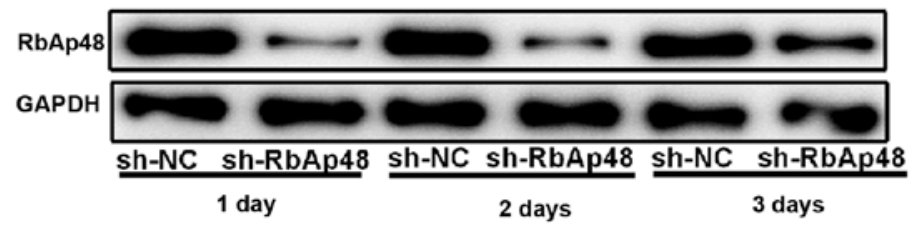

B
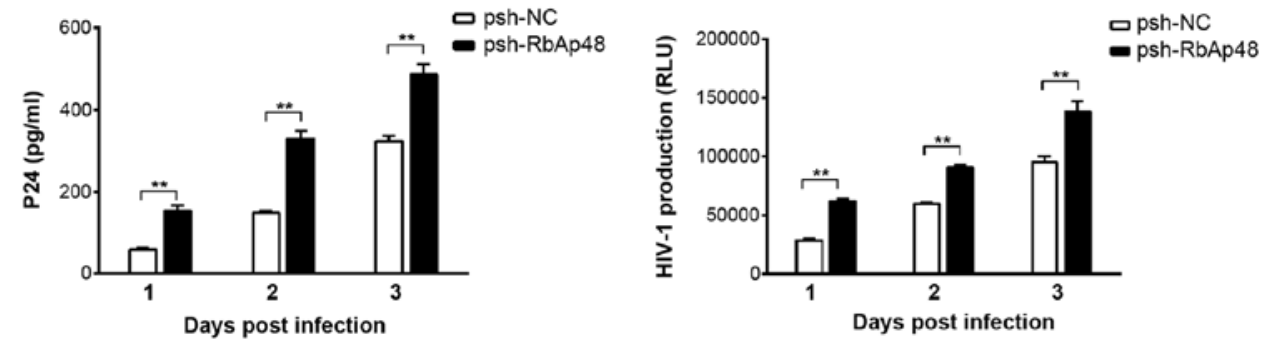

C
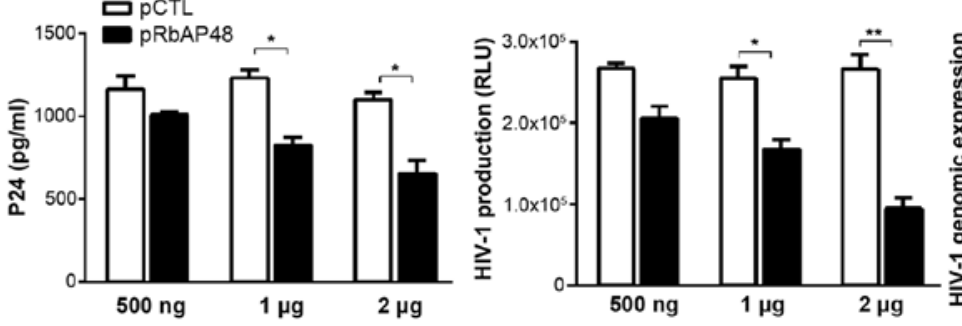

D
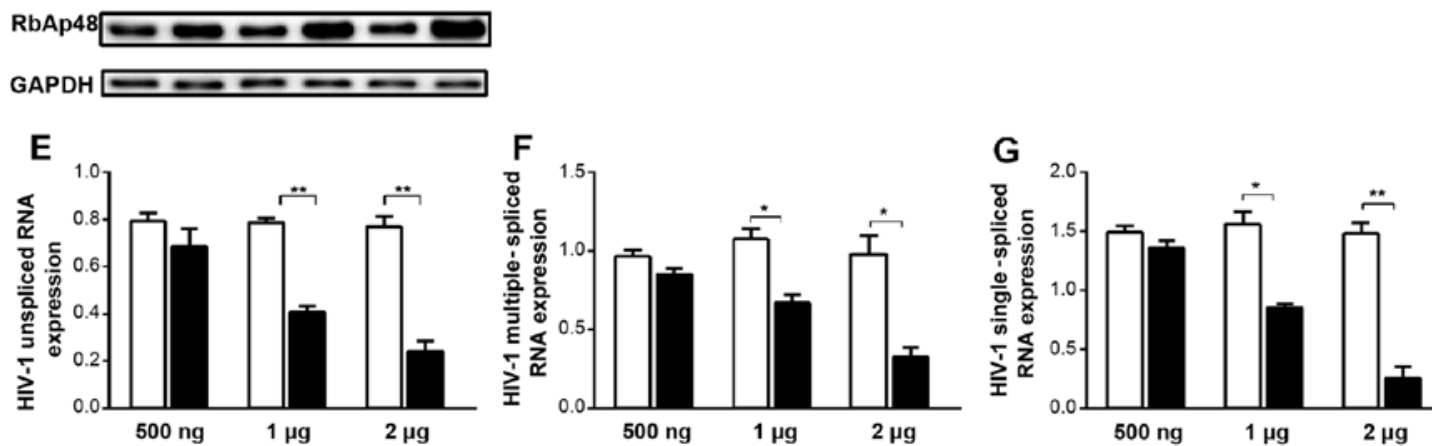

Figure 2. Retinoblastoma binding protein 4 (RbAp48) inhibits HIV-1 expression at the transcriptional level. (A) CEM-ss cells were transfected with $1 \mu \mathrm{g}$ sh-RbAp48 or sh-NC, respectively, followed by HIV-1 infection at $24 \mathrm{~h}$ post-transfection. RbAp48 protein expression after infection was detected by western blot analysis. (B) Production of viral particles was detected by HIV-1 P24 enzyme-linked immunosorbent assay (ELISA) kit (left panel). Viral particles were further quantified by infecting TZM-bl indicator cells and measuring the luciferase activity (right panel). (C) $293 \mathrm{~T}$ cells were transfected with $1.5 \mu \mathrm{g}$ of pNL4-3 along with increasing amounts of pRbAp48, with the supernatants being harvested 2 days post-transfection to analyse p24 antigen (left panel) and levels of infectious HIV-1 particles (right panel). (D-G) The same aliquots of 293T cells used in panel C were analysed by qPCR to determine HIV-1 genomic expression, unspliced, multiple-spliced and single-spliced HIV-1 RNA, respectively. ${ }^{*} \mathrm{P}<0.05$ and ${ }^{* * *} \mathrm{P}<0.01$.

RbAp48 represses HIV-1 promoter activity. We hypothesized that the inhibition of HIV-1 transcription observed in the presence of RbAp48 may result from a decrease in viral promoter activity. To futher explore this issue, 293T cells were co-transfected with a pGL3-LTR-Luc plasmid and pRbAp48 or pCTL. The expression of LTR activity in the cells transfected with the pRbAp48 was reduced by $39-71 \%$ compared with that in the $293 \mathrm{~T}$ cells that were transfected with the empty vector, in a dose-dependent manner (Fig. 3A). Conversely, knockdown of RbAp48 using a shRNA encoding vector resulted in a significant increase in basal transcription. There was a 1.37- to 1.97-fold increase in basal promoter activity upon knockdown of RbAp48 (Fig. 3B). We next evaluated whether RbAp48 affected the activation of LTR activity by exogenous stimuli, namely TNF- $\alpha$ and PMA. The results showed that overexpression of RbAp48 was capable of significantly overriding
TNF- $\alpha$ - and PMA-mediated transactivation of LTR both in TZM-bl cells with integrated LTR and 293T cells with transfected LTR-Luc (Fig. 3C and D). The inhibition of RbAp48 in TZM-bl cells without stimulation was moderate.

RbAp48 binds to the HIV-1 LTR. In light of the regulatory role of RbAp48 in the transcriptional process, we designed reporter assays to examine the possible response elements of RbAp48 on the HIV-1 LTR promoter. As shown in Fig. 4B, removal of modulatory and part of the enhancer region of the full length LTR [pGL3-LTR (-101/+181)-Luc] results in a 66.7\% decrease in luciferase activity by RbAp48 overexpression. Further deletion of the region that includes the $\mathrm{E} 2 \mathrm{~F} 1$ binding site [pGL3-LTR (-93/+181)-Luc] causes a 34\% decrease in LTR activity by RbAp48. The reduction in RbAp48 inhibition efficiency suggests that a candidate regulator in the region $(-101$ 


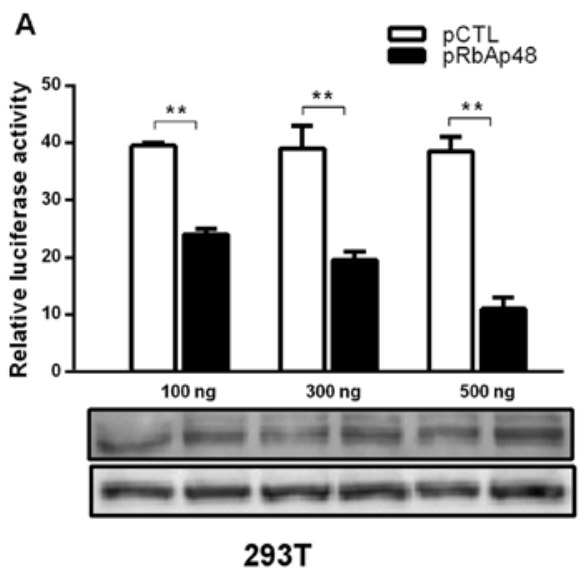

C

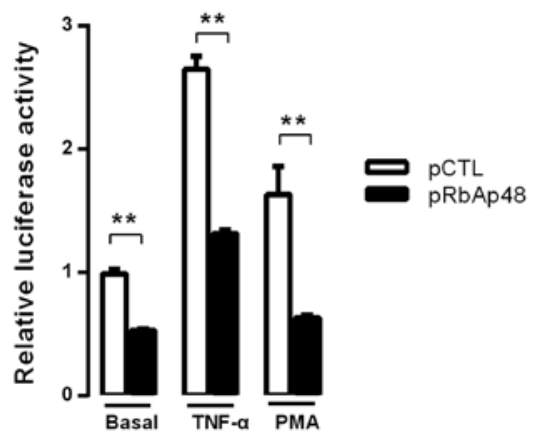

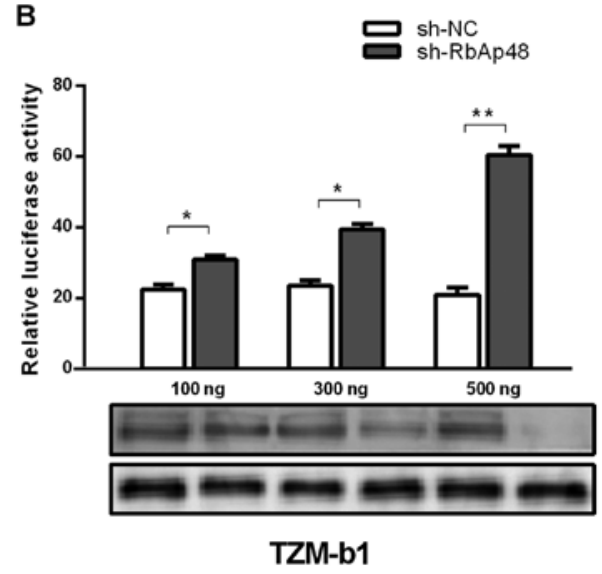

D

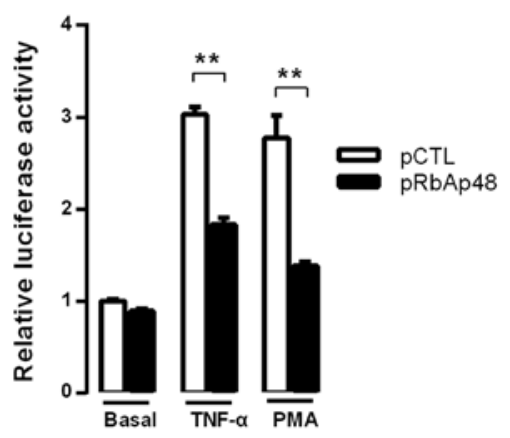

Figure 3. Retinoblastoma binding protein 4 (RbAp48) represses long term repeat (LTR) activity. (A and B) Transfection was performed with 80 ng pGL3-LTRLuc (Luciferase) and increasing amounts of vectors. Two days after transfection, the cells were analysed for endogenous RbAp48 expression by western blot analysis (lower panels) and firefly luciferase activity. (C and D) Firefly luciferase activity was detected in 293T cells and TZM-bl cells stimulated by TNF- $\alpha$ and phorbol 12-myristate 13-acetate (PMA) for $24 \mathrm{~h}$. The experiment was repeated three times and the results are shown as the fold of activation observed in the pCTL control condition, which was normalized to $1 .{ }^{*} \mathrm{P}<0.05$ and ${ }^{* *} \mathrm{P}<0.01$.

to -93) affects RbAp48 activity. However, when the enhancer region was deleted to leave a reporter construct containing only the core region of the promoter [pGL3-LTR $(-39 /+181)-L u c]$, RbAp48-mediated inhibition of transcriptional activity disappeared, raising the possibility that the -93 to -39 region, which contains the second $\mathrm{\kappa B}$ binding sites, includes candidate regulators which affect the ability of RbAp48 to repress LTR.

We also assessed the binding of RbAp48 to the NF- $\mathrm{kB}$ DNA-binding region in the LTR using EMSA (Fig. 4C). A shift was detected in the reaction between the probes and the nuclear extracts (lane 5). By contrast, TNF- $\alpha$ stimulation promoted complex formation (lanes 3,4$)$. When the 40 -fold excess cold selfcompetitor and mutated probe were used, complex formation was markedly decreased (lanes 2,7). A supershift was detected when an RbAp48 antibody was added to the reaction between probes and nuclear extracts (lane 6). In order to further examine whether RbAp48 binds to the HIV-1 LTR, TZM-bl cells harbouring integrated LTR were analyzed by ChIP assay using an anti-RbAp48 antibody. The results showed that RbAp48 was enriched in the LTR (Fig. 4D), and in parallel, we did not detect the binding of RbAp48 to the housekeeping GAPDH gene. Taken together, these results show that RbAp48 binds to the HIV-1 LTR.

\section{Discussion}

In the life cycle of HIV-1, transcription from the provirus is an important step for virus replication (26). The HIV-1 LTR controls the initiation of transcription through recruiting numerous cellular factors. Many cellular factors have been reported to directly bind to and regulate HIV-1 5' LTR promoter activity. Human factors YY1 and LSF inhibit HIV-1 LTR through the recruitment of HDAC1 (27). Nuclear matrix protein SMAR1 suppresses HIV-1 LTR-initiated transcription through chromatin remodelling (28). HIV-1 LTR-mediated transcription is also repressed by KRAB-zinc finger protein ZBRK1 through TRIM28 binding (19). However, there is limited information regarding transcription cofactors and their effects on LTR. In the present study, we have shown that RbAp48, a histone chaperone, is upregulated by HIV-1 infection. Knockdown of RbAp48 markedly increases the production of HIV-1 particles. Experiments to probe the step of viral replication blocked by $\mathrm{RbAp} 48$ revealed a substantial reduction in the synthesis of specific types of spliced HIV-1 RNA in infected cells. In general, the anti-viral host factors are always found downregulated following infection; however, several reports have shown that some anti-HIV molecules are upregulated. For example, the molecule caveolin-1 (Cav-1) suppresses HIV-1 replication and HIV-1 infection induces nuclear factor (NF)-IB expression, which in turn negatively impacts viral replication $(29,30)$. Hence, we hypothesized that RbAp48 may play a role in establishing HIV-1 infection and it represses viral gene expression in a feedback loop in a similar manner to Cav-1 and NF-IB.

Further assays to explore mechanisms responsible for the effects of RbAp48 in HIV-1 repression suggested that RbAp48 represses firefly luciferase activity driven by LTR both in the basal state as well as in the PMA- or TNF- $\alpha$-stimulated 
A

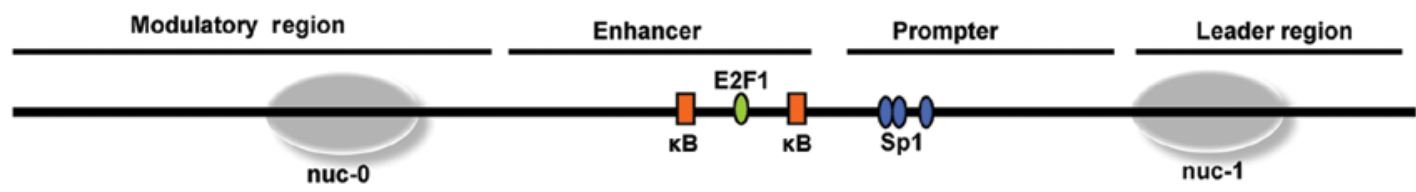

B
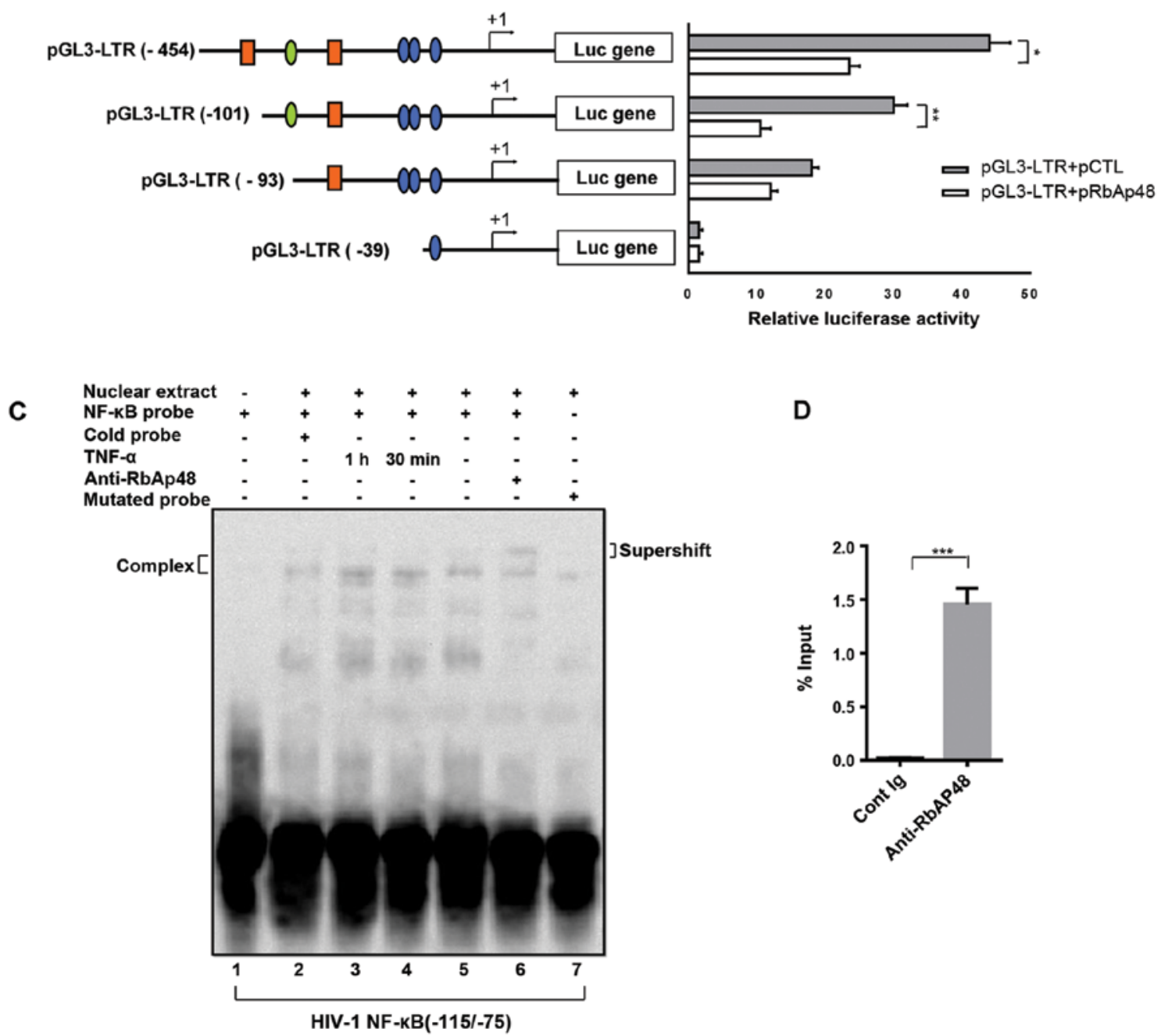

D

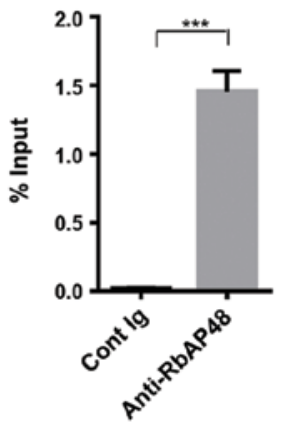

Figure 4. Retinoblastoma binding protein 4 (RbAp48) binds to the HIV-1 long term repeat (LTR). (A) Schematic of transcription factor binding sites in the HIV-1 LTR. (B) 293T cells were transfected with pRbAp48 or pCTL together with wild-type pGL3-LTR-Luc (Luciferase) or its corresponding deletion mutants. Transfected cells were harvested at two days after transfection and the luciferase activity was quantified. (C) Electrophoretic mobility shift assay showing the binding of RbAp48 to HIV-1 LTR. The 40bp full-length biotin-labelled NF- $\mathrm{\kappa B}$ region (-115/-75) from HIV-1 pNL4-3 was used as a probe. Approximately $120 \mathrm{fmol}$ DNA probes were incubated with $10 \mu \mathrm{g}$ of nuclear extracts. (D) Chromatin immunoprecipitation analysis (ChIP) experiments were performed with RbAp48 antibody in TZM-bl cells with integrated LTR. Occupancy of RbAp48 in the HIV-1 NF- $\mathrm{BB}$ region was measured by qPCR. Input DNA was used to normalize the amount of immunoprecipitated DNA. ${ }^{*} \mathrm{P}<0.05,{ }^{* *} \mathrm{P}<0.01$ and ${ }^{* * * *} \mathrm{P}<0.001$.

state in 293T cells. However, as the LTR-luciferase reporter gene is integrated in TZM-bl cells with a stable chromatin structure, LTR-driven luciferase activity was unaffected by exogenous RbAp48. When TZM-bl cells were stimulated with PMA and TNF- $\alpha$, which are known triggers of LTR and usually used to activate the NF- $\kappa \mathrm{B}$ signal pathway (31), exogenous RbAp48 significantly repressed luciferase activity. This suggests that RbAp48 may inhibit the activation of LTR activity by NF- $\kappa \mathrm{B}$ in a chromatin environment. This prompted us to perform a deletion strategy centered around two NF- $\mathrm{NB}$ binding sites of the HIV-1 LTR. The results indicated that the -101 to -93 region including the E2F1 binding site may contain an element which facilitates the repression activity of RbAp48, as the inhibitory effect of RbAp48 was markedly decreased in pGL3-LTR (-93/+181)-Luc. This prompted us to consider earlier studies indicating that E2F1 represses HIV-1 transcription through the NF- $\mathrm{BB}$ enhancer region of the HIV-1 LTR $(32,33)$. Furthermore, the main elements responsible for the regulation of RbAp48 may be located in the -93 to -39 region which contains the second $\kappa \mathrm{B}$ binding sites, as the inhibitory activity of RbAp48 was completely eliminated in this region. It has been previously demonstrated that $\mathrm{NF}-\kappa \mathrm{B}$ p50 represses the initiation of HIV transcription and facilitates HIV latency through HDAC recruitment (34). Additionally, RbAp48 belongs to the HDAC complex which is involved in transcriptional repression (14). This interaction suggests that the inhibitory function of RbAp48 may be associated with $\mathrm{NF}-\kappa \mathrm{B}$ p50 and HDAC. The results from gel shift assays showed that TNF- $\alpha$ stimulation promoted the binding of RbAp48 to LTR, which indicates that NF- $\kappa \mathrm{B}$ is implicated in the function of RbAp48. RbAp48 is usually studied 
as a component of chromatin remodelling factors. For example, as $\mathrm{RbAp} 48$ is present in NuRD, nucleosome-remodelling factor (NURF), Sin3A and polycomb repressive complex (PRC), $\mathrm{RbAp} 48$ therefore facilitates chromatin remodelling and organization $(6,35)$, and is dynamically recruited to the viral promoter; thus, it is possible that $\mathrm{RbAp} 48$ selectively recruits complexes to HIV-1 LTR, providing a beneficial chromatin environment for transcriptional repression.

Taken together, these findings indicate for the first time, to the best of our knowledge, that RbAp48 is a novel cofactor in HIV-1 infection and it may be described as a transcriptional inhibitor. Further analysis of the mechanism responsible for RbAp48 inhibition of HIV transcription may prompt the generation of specific therapies targeting HIV-1 latency.

\section{Acknowledgements}

We thank members of the Wu Laboratory for critical reading of the manuscript. The present study was supported by grants from the National Natural Science Foundation of China (grant no. 81273313), the National Key Projects of China (grant no. 2012ZX10001-003-004) and the Natural Science Funds of Zhejiang Province (grant no. LY14H190001).

\section{References}

1. Mohammadi P, di Iulio J, Muñoz M, Martinez R, Bartha I, Cavassini M, Thorball C, Fellay J, Beerenwinkel N, Ciuffi A and Telenti A: Dynamics of HIV latency and reactivation in a primary CD4 ${ }^{+} \mathrm{T}$ cell model. PLoS Pathog 10: e1004156, 2014.

2. Mbonye U and Karn J: Transcriptional control of HIV latency: cellular signaling pathways, epigenetics, happenstance and the hope for a cure. Virology 454-455: 328-339, 2014.

3. Maldarelli F, Wu X, Su L, Simonetti FR, Shao W, Hill S, Spindler J, Ferris AL, Mellors JW, Kearney MF, et al: HIV latency Specific HIV integration sites are linked to clonal expansion and persistence of infected cells. Science 345: 179-183, 2014.

4. Van Lint C, Bouchat S and Marcello A: HIV-1 transcription and latency: an update. Retrovirology 10: 67, 2013.

5. Qian YW, Wang YCJ, Hollingsworth RE Jr, Jones D, Ling N and Lee EYHP: A retinoblastoma-binding protein related to a negative regulator of Ras in yeast. Nature 364: 648-652, 1993.

6. Zhang W, Tyl M, Ward R, Sobott F, Maman J, Murthy AS, Watson AA, Fedorov O, Bowman A, Owen-Hughes T, et al: Structural plasticity of histones $\mathrm{H} 3-\mathrm{H} 4$ facilitates their allosteric exchange between RbAp48 and ASF1. Nat Struct Mol Biol 20: 29-35, 2102, 2013

7. Loyola A and Almouzni G: Histone chaperones, a supporting role in the limelight. Biochim Biophys Acta 1677: 3-11, 2004.

8. Allen HF, Wade PA and Kutateladze TG: The NuRD architecture. Cell Mol Life Sci 70: 3513-3524, 2013.

9. Zhang Y, Ng HH, Erdjument-Bromage H, Tempst P, Bird A and Reinberg D: Analysis of the NuRD subunits reveals a histone deacetylase core complex and a connection with DNA methylation. Genes Dev 13: 1924-1935, 1999.

10. Cismasiu VB, Paskaleva E, Suman Daya S, Canki M, Duus K and Avram D: BCL11B is a general transcriptional repressor of the HIV-1 long terminal repeat in T lymphocytes through recruitment of the NuRD complex. Virology 380: 173-181, 2008.

11. O'Connor MD, Wederell E, Robertson G, Delaney A Morozova O, Poon SSS, Yap D, Fee J, Zhao Y, McDonald H, et al: Retinoblastoma-binding proteins 4 and 9 are important for human pluripotent stem cell maintenance. Exp Hematol 39: 866-79.e1, 2011.

12. Creekmore AL, Walt KA, Schultz-Norton JR, Ziegler YS, McLeod IX, Yates JR and Nardulli AM: The role of retinoblastoma-associated proteins 46 and 48 in estrogen receptor $\alpha$ mediated gene expression. Mol Cell Endocrinol 291: 79-86, 2008.

13. Ishimaru N, Arakaki R, Yoshida S, Yamada A, Noji S and Hayashi Y: Expression of the retinoblastoma protein RbAp48 in exocrine glands leads to Sjögren's syndrome-like autoimmune exocrinopathy. J Exp Med 205: 2915-2927, 2008.
14. Nicolas E, Morales V, Magnaghi-Jaulin L, Harel-Bellan A, Richard-Foy $\mathrm{H}$ and Trouche D: RbAp48 belongs to the histone deacetylase complex that associates with the retinoblastoma protein. J Biol Chem 275: 9797-9804, 2000.

15. Wang J, Lv HJ, Xu L, Yang J, Yang ZX and Wu NP: Generation of human immunodeficiency virus-1 long terminal repeat reporter genes by rapid polymerase chain reaction-nediated mutagenesis. Lab Med 44: 220-227, 2013.

16. Liu L, Wang W, Yang L, Ren H, Kimata JT and Zhou P: Trimeric glycosylphosphatidylinositol-anchored HCDR3 of broadly neutralizing antibody PG16 is a potent HIV-1 entry inhibitor. J Virol 87: 1899-1905, 2013.

17. Yang J, Yang Z, Lv H, Lou Y, Wang J and Wu N: Bridging HIV-1 cellular latency and clinical long-term non-progressor: an interactomic view. PLoS One 8: e55791, 2013.

18. O'Doherty U, Swiggard WJ and Malim MH: Human immunodeficiency virus type 1 spinoculation enhances infection through virus binding. J Virol 74: 10074-10080, 2000.

19. Nishitsuji H, Abe M, Sawada R and Takaku H: ZBRK1 represses HIV-1 LTR-mediated transcription. FEBS Lett 586: 3562-3568, 2012.

20. Casabianca A, Gori C, Orlandi C, Forbici F, Federico Perno C and Magnani M: Fast and sensitive quantitative detection of HIV DNA in whole blood leucocytes by SYBR green I real-time PCR assay. Mol Cell Probes 21: 368-378, 2007.

21. Jablonski JA and Caputi M: Role of cellular RNA processing factors in human immunodeficiency virus type 1 mRNA metabolism, replication, and infectivity. J Virol 83: 981-992, 2009.

22. Yedavalli VSRK and Jeang KT: Trimethylguanosine capping selectively promotes expression of Rev-dependent HIV-1 RNAs Proc Natl Acad Sci USA 107: 14787-14792, 2010.

23. Lejon S, Thong SY, Murthy A, AlQarni S, Murzina NV, Blobel GA, Laue ED and Mackay JP: Insights into association of the NuRD complex with FOG-1 from the crystal structure of an RbAp48.FOG-1 complex. J Biol Chem 286: 1196-1203, 2011.

24. Lewin SR and Rouzioux C: HIV cure and eradication: how will we get from the laboratory to effective clinical trials? AIDS 25 : 885-897, 2011.

25. Gururaja T, Li W, Bernstein J, Payan DG and Anderson DC: Use of MEDUSA-based data analysis and capillary HPLC-ion-trap mass spectrometry to examine complex immunoaffinity extracts of RBAp48. J Proteome Res 1: 253-261, 2002.

26. Kumar A, Darcis G, Van Lint C and Herbein G: Epigenetic control of HIV-1 post integration latency: implications for therapy. Clin Epigenetics 7: 103, 2015.

27. Coull JJ, Romerio F, Sun JM, Volker JL, Galvin KM, Davie JR, Shi Y, Hansen U and Margolis DM: The human factors YY1 and LSF repress the human immunodeficiency virus type 1 long terminal repeat via recruitment of histone deacetylase 1. J Virol 74: 6790-6799, 2000.

28. Sreenath K, Pavithra L, Singh S, Sinha S, Dash PK, Siddappa NB, Ranga U, Mitra D and Chattopadhyay S: Nuclear matrix protein SMAR1 represses HIV-1 LTR mediated transcription through chromatin remodeling. Virology 400: 76-85, 2010.

29. Wang XM, Nadeau PE, Lin S, Abbott JR and Mergia A: Caveolin 1 inhibits HIV replication by transcriptional repression mediated through NF-кB. J Virol 85: 5483-5493, 2011.

30. Vemula SV, Veerasamy R, Ragupathy V, Biswas S, Devadas K and Hewlett I: HIV-1 induced nuclear factor I-B (NF-IB) expression negatively regulates HIV-1 replication through interaction with the long terminal repeat region. Viruses 7: 543-558, 2015.

31. Rom S, Reichenbach NL, Dykstra H and Persidsky Y: The dual action of poly(ADP-ribose) polymerase -1 (PARP-1) inhibition in HIV-1 infection: HIV-1 LTR inhibition and diminution in Rho GTPase activity. Front Microbiol 6: 878, 2015.

32. Kundu M, Srinivasan A, Pomerantz RJ and Khalili K: Evidence that a cell cycle regulator, E2F1, down-regulates transcriptional activity of the human immunodeficiency virus type 1 promoter. J Virol 69: 6940-6946, 1995.

33. Kundu M, Guermah M, Roeder RG, Amini S and Khalili K: Interaction between cell cycle regulator, E2F-1, and NF-kappaB mediates repression of HIV-1 gene transcription. J Biol Chem 272: 29468-29474, 1997.

34. Williams SA, Chen LF, Kwon H, Ruiz-Jarabo CM, Verdin E and Greene WC: NF-kappaB p50 promotes HIV latency through HDAC recruitment and repression of transcriptional initiation. EMBO J 25: 139-149, 2006.

35. Jullien PE, Mosquna A, Ingouff M, Sakata T, Ohad N and Berger F: Retinoblastoma and its binding partner MSI1 control imprinting in Arabidopsis. PLoS Biol 6: e194, 2008. 